Moore, W. B. \& RaInbow, C. (1955). J. gen. Microbiol. 13, 190-197.

\title{
Nutritional Requirements and Biochemical Activities of Brewery Lactobacilli
}

\author{
By W. B. MOORE AND C. RAINBOW \\ Department of Applied Biochemistry, University of Birmingham
}

\begin{abstract}
SUMMARY: Four strains of lactobacilli, isolated from the contaminant flora of English top fermentation brewers' yeasts, were found to be heterofermentative. Two strains were tentatively identified as Lactobacillus brevis. For growth, all the strains required exogenous supplies of a number of growth factors of the vitamin $B$ complex, a pyrimidine, at least one purine and at least 14 amino acids. The ability of hypoxanthine to substitute for both adenine and guinane in one strain of $L$. brevis is especially notable. L-Arginine, essential for the growth of all strains, increased the rate and final amount of growth in glucose medium of both strains of $L$. brevis, when it was supplied in relatively high concentration. Suspensions of the organisms converted arginine into ornithine, ammonia and a small amount of citrulline. Maltose, fructose and L-arabinose were better substrates for the growth of all strains than glucose, which, as major source of energy, supported little or no growth of the two unidentified strains. All the strains transformed fructose into mannitol. The rate and extent of growth of most strains on hexoses and the rate of growth of all strains on maltose were increased by the presence of acetate. Whereas the strains of $L$. brevis formed only little acid from glucose, all strains produced considerable amounts of volatile and non-volatile acids from $L$-arabinose.
\end{abstract}

Certain lactic acid bacteria have long been recognized as beer spoilage organisms. However, unlike their counterparts which inhabit dairy products there have been few studies of the nutrition of these bacteria. Recently, Russell, Bhandari \& Walker (1954) surveyed the vitamin requirements of 34 strains, comprising 10 species of brewery lactic acid bacteria. The present work is restricted to four strains isolated from pitching yeasts of top fermentation breweries, and reports on their nutritional requirements with respect to growth factors of the vitamin $\mathbf{B}$ complex, purines, pyrimidines, amino acids and major sources of energy, and some biochemical activities.

\section{METHODS}

Organisms. The strains used in this work were isolated from the contaminant flora of samples of English top fermentation yeasts obtained from three different breweries. Stock cultures were carried on liquid unhopped malt wort. The strains were designated L3, L4, L5 and L 6 .

Media. (a) Unhopped malt wort (sp.gr. 1·036) was prepared from brewers' pale ale malt. (b) The complete medium for strains $\mathrm{L} 3$ and $\mathrm{L} 4$ was as described by Chamberlain \& Rainbow (1954) for Lactobacillus brevis, with the addition of $1 \mathrm{mg}$. each of adenine and guanine $/ 100 \mathrm{ml}$; f for convenience this will be denoted as CR medium. This CR medium was also used for strains L5 and L6, but the glucose was omitted and $1 \cdot 8 \mathrm{~g}$. L-arabinose added. Except where 
otherwise stated, media and test additions were sterilized in admixture by heating in the autoclave momentarily to $15 \mathrm{lb}$./sq.in. pressure.

Inocula. (a) For tube tests, transfers were made from stock wort cultures into $6 \mathrm{ml}$. of appropriate complete semi-defined medium. After incubation at $28^{\circ}$ for 16-24 hr., the cells were harvested by centrifugation, washed three times with portions each of $6 \mathrm{ml}$. of sterile $0.85 \%$ saline and resuspended in a further $6 \mathrm{ml}$. of sterile saline. A portion of this suspension was diluted 150 times with sterile saline and after shaking well, one drop of this dilute suspension was used to inoculate each tube.

(b) For work with suspensions of organisms, inocula were prepared by transferring from stock cultures to $6 \mathrm{ml}$. portions of malt wort medium, incubating at $32^{\circ}$ and washing the crop three times as above. The washed organisms were then suspended in the appropriate solutions for metabolic study.

(c) Inocula for larger cultures $(60 \mathrm{ml}$.) in malt wort were grown as in (b) and for those in CR media as in $(a)$. Each $60 \mathrm{ml}$. culture was inoculated with $3 \mathrm{ml}$. of the resultant subculture, without washing (for malt wort cultures) or after washing three times and resuspending as in $(a)$ (for cultures in CR medium).

Tube tests (6 ml. cultures) for growth response were carried out as described by Chamberlain \& Rainbow (1954).

Experiments with suspensions of organisms were carried out at $28^{\circ}$, the substances to be metabolized being added as sterile aqueous solutions at pH 6.5, to organisms which had been harvested and washed as described above. After incubation, the organisms were centrifuged and the supernatant fluids examined by paper chromatography or by other procedures as described.

Determination of fermentation acids. After incubation for $120 \mathrm{hr}$., the total volatile acids formed in $60 \mathrm{ml}$. cultures were determined as described by Fred, Peterson \& Davenport (1919). Total acids were determined either by direct titrations of measured samples of the fermented liquid or, when fermentation was carried out in the presence of calcium carbonate, by determination as calcium oxalate of the calcium in solution. The figures for non-volatile acid were obtained by difference.

Paper chromatography. The appropriate supernatant fluids were examined by single run descending papergrams. The upper layer of a mixture of $n$ butanol + ethanol + water $(4: 1: 5, \mathrm{v} / \mathrm{v})$ was used to separate fructose from mannitol and the components were revealed by spraying with $\mathrm{AgNO}_{3}$ reagent (Hough, 1950). For runs involving arginine, the conditions and solvents were those used by Partridge (1948), amino acids being detected by spraying with $0.1 \%$ ninhydrin in $n$-butanol saturated with water.

\section{RESULTS}

\section{Characteristics of the strains}

Morphological. Each isolate consisted of non-motile, non-sporing, Grampositive rods.

Cultural. The strains grew copiously in unhopped malt wort, producing a 
silky turbidity which settled after extended incubation to a powdery deposit. Good growth also occurred in stab cultures in malt agar.

Temperature and $\mathrm{pH}$. Tube tests showed that all strains grew well in malt wort at $\mathrm{pH} 5 \cdot 8$ and $32-35^{\circ}$ and in appropriate semi-defined medium at pH $6 \cdot 2$ and $26-28^{\circ}$.

Biochemical. All the isolates were catalase-negative, vP-negative and produced lactic acid when grown on malt wort. Lactic acid was isolated by the method of Franzen \& Stern (1921). Polarimetric examination of the ethereal extracts of the free acid and the behaviour of the water of crystallization on heating the crystalline zinc salt showed that DL-lactic acid was formed in each case. Other biochemical activities of the strains are reported below.

Identification. Strains $\mathrm{L} 3$ and $\mathrm{L} 4$ are tentatively identified as strains of Lactobacillus brevis, and strains L5 and L6 may also belong to the same species, differing from $\mathrm{L} 3$ and $\mathrm{L} 4$ especially with regard to capacity to utilize glucose for growth (see below).

\section{Nutritional requirements}

Growth factors, pyrimidines and purines. The requirements of the strains for individual growth factors of the vitamin $\mathbf{B}$ complex and for pyrimidines were determined by growth tests in which each test substance was omitted in turn from an otherwise complete cr medium. To determine the purine requirements, growth tests were carried out in purine-free $\mathrm{CR}$ medium, the purines (adenine, hypoxanthine, guanine and xanthine) being added singly and in all possible combinations.

\section{Table 1. Growth factor requirements of brewery lactobacilli}

Growth tests were made at $28^{\circ}$ in $\mathrm{CR}$ medium containing $1 \%$ each of glucose and L-arabinose. Observations were qualitatively the same over the incubation period of $48-148 \mathrm{hr}$. The result of omission of a growth factor is signified as follows: $\mathbf{E}$ (essential)=little or no growth; $\mathbf{S}$ (stimulatory)= growth restricted to $50 \%$ or less of maximum growth; $\mathrm{O}$ (not essential) $=$ little or no restriction of growth.

\begin{tabular}{|c|c|c|c|c|}
\hline Growth factor ... & $\ldots$ & L3 & L4 & L5 \\
\hline Thiamine & & $\mathrm{s}$ & $\mathbf{S}$ & $\mathbf{S}$ \\
\hline Pyridoxin & & o & o & o \\
\hline Pantothenate & & $\mathbf{E}$ & $\mathbf{E}$ & $\mathbf{E}$ \\
\hline Nicotinate & & $\mathbf{E}$ & $\mathbf{E}$ & $\mathbf{E}$ \\
\hline Riboflavin & & $\mathbf{E}$ & O & $\mathbf{O}$ \\
\hline Biotin & & $\mathbf{S}$ & $\mathbf{s}$ & $\mathbf{s}$ \\
\hline Pteroylglutamic acid & & $\mathbf{E}$ & $\mathbf{E}$ & $\mathbf{E}$ \\
\hline Uracil & & $\mathbf{E}$ & $\mathbf{E}$ & $\mathbf{E}$ \\
\hline Purines & & $\mathbf{E}$ & $\mathbf{E}$ & $\mathbf{E}$ \\
\hline
\end{tabular}

Table 1 summarizes the results. All strains showed absolute requirements for pantothenate, nicotinate, pteroylglutamate (PGA), a purine and uracil. In addition, the growth of all strains was severely restricted by omission of thiamine or biotin. For strains L3, L5 and L6, cytosine or orotic acid, but not thymine, could replace uracil. For strain L 4, uracil was replaceable, only after a period of induction, by cytosine, but not by orotic acid or thymine. The 
purine requirement of strains $\mathrm{L} 3, \mathrm{~L} 5$ and $\mathrm{L} 6$ was met by either adenine, hypoxanthine, guanine or xanthine. However, strain L 4 was unusual in that its requirement for two purines (adenine and guanine or xanthine) could be met by hypoxanthine alone. This fact, which was applied in another connexion by Chamberlain \& Rainbow (1954), suggests that hypoxanthine is a common precursor of both adenine and guanine in strain L 4. The general pattern of growth factor requirements was similar for all strains, but strain L3 was unique in requiring added riboflavin, to which it responded sharply over the range $0-1.5 \mathrm{~m} \mu \mathrm{g} . / \mathrm{ml}$.

Note on casein hydrolysate. The casein hydrolysate in the CR medium used for the above tests was manifestly free from pantothenate, nicotinate, PGA, riboflavin, uracil and purines. Tests with other suitable micro-organisms showed that the casein hydrolysate was also substantially free from thiamine, biotin, pyridoxin and $p$-aminobenzoate, although it is recognized that the preparation may still contain quantities of these factors (in particular, of biotin) sufficient to permit partial or full growth of strains L3, L 4, L5 and L6.

Amino acids. To determine the requirements of each strain for individual amino acids, growth tests were carried out for $4-5$ days at $28^{\circ}$ in $\mathrm{CR}$ medium, the casein hydrolysate of which was replaced by a mixture of 18 amino acids (Henderson \& Snell, 1948) each of which could be omitted in turn from the otherwise complete medium. The amino acids used in these tests were DLisomers except as stated below.

The results may be summarized as follows:

(a) Essential in all cases: L-arginine, L-aspartate, L-glutamate, isoleucine, L-leucine, phenylalanine, L-proline, serine, threonine, tryptophan and valine.

(b) Alanine, glycine, L-histidine, L-lysine, methionine and L-tyrosine were either essential or markedly stimulated the growth of all strains, except as recorded in $(c)$ below.

(c) Not essential: for strain L3, alanine and L-histidine; for strain L4, L-lysine and L-histidine.

Utilization of carbohydrates for growth. The capacity of a number of sugars, sugar alcohols and acetate to support growth was determined by tube tests in which the test substance was added individually to $\mathrm{CR}$ medium devoid of carbohydrate and acetate. The following results (Table 2) are noteworthy:

(a) Mannose, $\mathrm{D}$-arabinose, sucrose, lactose, raffinose and acetate did not promote the growth of any strain and sorbitol and mannitol were at best only feebly utilized.

(b) Glucose was not utilized for growth by strains L5 and L6 as a sole source of carbon and/or energy, and although strains L 3 and L4 grew on it, growth (especially during the first $48 \mathrm{hr}$.) was inferior to that on maltose, fructose or L-arabinose, all three of which were utilized by all strains.

(c) Galactose was utilized for growth, albeit poorly, by all strains.

These tests were repeated in the presence of $32 \mathrm{mg}$. $\mathrm{CH}_{3} \mathrm{COONa}_{3} 3 \mathrm{H}_{2} \mathrm{O} / \mathrm{ml}$. The following effects of acetate were observed. Growth of strains L 3 and L4 on glucose and on galactose, and of strain L4 on fructose, was enhanced in the early stages by the presence of acetate. The final amount of growth of all 
strains on L-arabinose or on glucose $+\mathrm{L}$-arabinose was lower in the presence of acetate. During the first $\mathbf{7 2} \mathrm{hr}$., the growth of all strains on maltose was strongly stimulated by acetate, but the final $(168 \mathrm{hr}$.) amount of growth was little affected. Indeed, the turbidity of cultures of L 3, L 4 and L 5 on maltose and acetate rose to a maximum in about 3 days, falling appreciably thereafter.

\section{Table 2. Utilization of carbohydrates for growth by brewery lactobacilli}

Growth tests were made at $28^{\circ}$ in basal $\mathrm{CR}$ medium free from carbohydrate and acetate. Test substances were added as follows (mg./ml.): hexoses and sugar alcohols, 10; pentoses, $8 \cdot 3$; disaccharides, $9 \cdot 5$; raffinose, 9.7 $; \mathrm{CH}_{3} \mathrm{COONa} .3 \mathrm{H}_{2} \mathrm{O}$ (adjusted to $\mathrm{pH} \mathrm{6 \cdot 2)}$ ), 32. The Spekker reading (168 hr.) of control tubes containing no test substance was 0.05 or less.

\begin{tabular}{|c|c|c|c|c|}
\hline \multirow{3}{*}{$\begin{array}{c}\text { Relative } \\
\text { degrees } \\
\text { of growth } \\
\text { (Spekker } \\
\text { readings) } \\
>\mathbf{1 . 2 0}\end{array}$} & \multicolumn{4}{|c|}{ Strain of lactobacillus } \\
\hline & \multicolumn{4}{|c|}{$\begin{array}{cc}\text { L4 } & \text { L5 } \\
\text { Additions to basal } \mathrm{CR} & \text { medium }\end{array}$} \\
\hline & Glucose $+\mathrm{L}$-arabinose & Glucose $+\mathrm{L}$-arabinose & L-Arabinose & - \\
\hline $0 \cdot 80-1 \cdot 20$ & Fructose or maltose & $\begin{array}{l}\text { Fructose, maltose, } \\
\text { L-arabinose, xylose } \\
\text { or ribose }\end{array}$ & $\begin{array}{l}\text { Ribose*, fructose or } \\
\text { maltose }\end{array}$ & $\begin{array}{l}\text { Fructose or glucose }+ \\
\text { L-arabinose }\end{array}$ \\
\hline $0.40-0.80$ & $\begin{array}{l}\text { L-Arabinose, glucose, } \\
\text { galactose, xylose or } \\
\text { ribose* }\end{array}$ & $\begin{array}{l}\text { Galactose* or } \\
\text { glucose* }\end{array}$ & $\begin{array}{l}\text { Glucose }+\mathbf{L} \text {-arabinose, } \\
\text { xylose or galactose }\end{array}$ & $\begin{array}{l}\text { L-Arabinose, maltose, } \\
\text { xylose* or ribose* }\end{array}$ \\
\hline $\begin{array}{l}0 \cdot 20-0 \cdot 40 \\
<0 \cdot 20\end{array}$ & $\begin{array}{l}\text { Sorbitol } \\
\text { Mannitol }\end{array}$ & Sorbitol or mannitol & $\begin{array}{l}\text { Mannitol or sorbitol } \\
\text { Glucose }\end{array}$ & $\begin{array}{l}\text { Galactose or sorbitol } \\
\text { Glucose or mannitol }\end{array}$ \\
\hline
\end{tabular}

Not utilized by any strain: mannose, D-arabinose, sucrose, lactose, raffinose, acetate

* Signifies growth only after a lag period of 3 or more days.

The rate of growth of all strains on pentoses appeared to depend on some chemical change, induced by thermal sterilization, during which a stimulatory substance or substances was produced by interaction between pentose and a constituent or constituents of the medium. Thus, the growth of all strains was more rapid when sterilization was carried out with pentose in admixture with the other medium constituents than when the pentose and the other constituents were sterilized separately and then mixed when cold.

\section{Biochemical activities}

Types of fermentation. The molecular ratios of non-volatile to volatile acid formed by each strain after growth in malt wort varied from 3.3 to 5.9 or from $4 \cdot 1$ to $4 \cdot 8$ when $\mathrm{CaCO}_{3}$ was present (Table 3). These values were low compared with those expected from homofermentative lactobacilli and indicated that our strains were heterofermentative. Strain L3 was selected for further examination, by similar means, for the type of fermentation effected in acetate-free $\mathbf{C R}$ media containing either glucose or L-arabinose. From glucose, only small amounts of acid were formed, the ratio of non-volatile to volatile acid being approximately 2:1. Much larger amounts of total acids, and especially of volatile acid, were produced from L-arabinose, the corresponding ratio being nearly 1:1 (Table 3; cf. Gest \& Lampen, 1952). 
Transformation of fructose. Cultures of strains L3, L4, L5 and L 6 in CR medium containing $1.8 \%$ fructose as sole carbohydrate, were grown at $28^{\circ}$ for $144 \mathrm{hr}$. The presence of mannitol in the culture fluids of all strains was demonstrated by paper chromatography. This was confirmed by growing strain L3 on $60 \mathrm{ml}$. CR medium (containing $1.8 \%$ fructose instead of glucose) for $144 \mathrm{hr}$. The method of Peterson \& Fred (1920) was used to isolate from the culture filtrate $125 \mathrm{mg}$. of a substance which resembled an authentic specimen of mannitol in melting point (found, $166^{\circ}$; authentic specimen, $167-168^{\circ}$; mixed melting point, $166-167^{\circ}$ ) and in chromatographic mobility.

\section{Table 3. Acid formation by strain L3}

$60 \mathrm{ml}$. cultures were grown for $120 \mathrm{hr}$. at $32^{\circ}$ (in malt wort) or at $28^{\circ}$ in $\mathrm{Cr}$ medium, devoid of acetate and containing either $5 \%$ glucose or $4.5 \% \mathrm{~L}$-arabinose. The ratio $(a) /(b)$ assumes that the acids concerned are monobasic.

\begin{tabular}{|c|c|c|c|c|}
\hline & & Acid fo & ned & \\
\hline & Total & $\begin{array}{c}\text { Non-volatile } \\
(a)\end{array}$ & $\begin{array}{l}\text { Volatile } \\
\qquad(b)\end{array}$ & $\begin{array}{l}\text { Ratio } \\
(a) /(b)\end{array}$ \\
\hline & & 1. $0 \cdot 10 \mathrm{~N}-\mathrm{acid} /$ & ml. cultu & \\
\hline Malt wort & $5 \cdot 76$ & $4 \cdot 93$ & $0 \cdot 83$ & $5 \cdot 9$ \\
\hline Malt wort $+\mathrm{CaCO}_{3}$ & $18 \cdot 22$ & $14 \cdot 81$ & $3 \cdot 41$ & $4 \cdot 3$ \\
\hline Glucose medium & $1 \cdot 25$ & $0 \cdot 83$ & $0 \cdot 42$ & $2 \cdot 0$ \\
\hline Arabinose medium & $11 \cdot 35$ & $5 \cdot 96$ & $5 \cdot 39$ & $1 \cdot 1$ \\
\hline
\end{tabular}

Transformation of arginine. The rate and final amount (after $168 \mathrm{hr}$.) of growth of strains L3 and L4 in CR medium containing glucose as sole carbohydrate was considerably increased by the addition of 1-2 mg. L-arginine $/ \mathrm{ml}$., but not by DL-citrulline and/or DL-ornithine. The stimulatory action of arginine was not apparent when L-arabinose was also present in the medium. When washed cell suspensions of strains $\mathrm{L} 3, \mathrm{~L} 4, \mathrm{~L} 5$ and $\mathrm{L} 6$ were allowed to metabolize solutions containing 4-8 $\mathrm{mg}$. L-arginine/ml. and the resultant supernatants were examined by paper chromatography, ninhydrin-positive substances identical in mobility with ornithine and citrulline were shown to be formed by all strains. The presence of citrulline, which was found only in small amounts, was confirmed by use of the $p$-dimethylaminobenzaldehyde spray of Dent (1948). The transformation of arginine by suspensions of organisms proceeded in the absence of glucose and growth factors and was accompanied by a change of $\mathrm{pH}$ from $\mathbf{6 \cdot 2}$ to $8 \cdot 6$; ammonia was detected by Nessler's reagent in the supernatant or in the distillate from the supernatant made alkaline with $\mathrm{KOH}$.

Dent's spray also serves to detect urea. Although $20 \mu \mathrm{g}$. urea was readily detected on the chromatograms by this method, the spot occupying a position between those of arginine and ornithine, no urea was detected in a portion of metabolized supernatant corresponding to $40 \mu \mathrm{g}$. L-arginine. Urea was also not detected by the xanthydrol test applied directly to the supernatants. No significant decomposition of urea was detected when washed organisms were incubated at $28^{\circ}$ for $20 \mathrm{hr}$. with urea solution (1 mg./ml.). 
That the substance formed from arginine was indeed ornithine and not lysine (which possesses the same chromatographic mobility under the conditions used) was confirmed by chromatography using the same $n$-butanol/ ethanol/water solvent, but carried out under the acid conditions created by the presence of $1: 1$ glacial acetic acid + water mixture in the cabinet. Under these conditions, the substance derived from arginine was distinct from lysine, and identical with authentic ornithine. By similar methods, suspensions of strain L3 were shown to convert citrulline partially to ornithine, whilst ornithine was not attacked.

\section{DISCUSSION}

The lactobacilli which inhabit diary products are recognized as nutritionally exacting organisms, particularly with respect to their requirements for exogenous amino acids, purines, pyrimidines and growth factors of the vitamin $\mathbf{B}$ complex. The present work shows that some actual or potential beer spoilage lactobacilli are equally fastidious in these respects. Whilst the general pattern of requirements for growth factors shown by our strains agrees with that found by Russell et al. (1954), our strains differ in their absolute requirement for pteroylglutamic acid which was not required by any of the strains of Russell et al.

Our strains are heterofermentative lactobacilli, forming little acid from glucose but considerable amounts of acid from L-arabinose. They show certain unusual preferences with respect to utilization of carbohydrate (presumably required as a source of energy). Thus, only two strains utilized glucose for growth, whilst all strains grew well on L-arabinose, fructose or maltose. It thus appears that growth on maltose does not involve preliminary hydrolysis of the maltose molecule to glucose. The availability of maltose may reflect a nice adaptation of these lactobacilli to their natural environment (beer) the predominant carbohydrate of which is maltose. It is also of possible evolutionary interest that all the strains are capable of utilizing galactose for growth, if only to a limited extent.

Apart from the function of L-arginine as an essential amino acid, the stimulation of growth in glucose medium of strains L3 and L 4 by relatively high concentrations of L-arginine suggests that the cleavage of the arginine molecule provides these organisms with a source of energy or some important intermediate, such as metabolic $\mathrm{CO}_{2}$, which may also be derived from L-arabinose since the stimulatory effect of arginine is not apparent when L-arabinose is present.

The transformation of $\mathbf{L}$-arginine to ornithine and ammonia by all strains is of interest in view of the reports of the arginine dihydrolase activities of other lactic acid bacteria (Hills, 1940; Oginsky \& Gehrig, 1952) and because the ability to effect this transformation has been suggested as a physiological test in the classification of lactobacilli (Briggs, 1953). Although inconclusive, our evidence favours the hypothesis that our lactobacilli transform arginine by the dihydrolase system. The alternative (Krebs) mechanism requires the formation of urea as an end product by cells which do not possess urease activity. 
The relatively feeble metabolism of citrulline to ornithine by suspensions of strain L3 accords with the observations of Oginsky \& Gehrig (1952) who showed that, although acetone-dried cells of Streptococcus faecalis formed ammonia and $\mathrm{CO}_{2}$ as rapidly from citrulline as from arginine, intact organisms effected only a slow breakdown of citrulline as compared with arginine.

We are indebted to the Department of Scientific and Industrial Research for a grant to one of us (W. B. M.) and to Dr Dora Kulka, who isolated the strains used in this investigation and identified the lactic acid produced by them.

\section{REFERENCES}

Briggs, M. (1953). The classification of lactobacilli by means of physiological tests. J. gen. Microbiol. 9, 234.

Chamberlain, N. \& Rainbow, C. (1954). The formation of diazotizable amine and hypoxanthine by a yeast: possible implications in the biosynthesis of purines. J. gen. Microbiol. 11, 180.

DENT, C. E. (1948). A study of the behaviour of some sixty amino-acids and other ninhydrin-reacting substances on phenol-'collidine' filter-paper chromatograms, with notes as to the occurrence of some of them in biological fluids. Biochem. J. 43, 169.

Franzen, H. \& STern, E. (1921). Über das Vorkommen von Äthylidenmilchsäure in den Blattern der Himbeere (Rubus Idaeus). Z. physiol. Chem. 115, 270.

Fred, E. B., Peterson, W. H. \& Davenport, A. (1919). Acid fermentation of xylose. J. biol. Chem. 39, 347.

Gest, H. \& Lampen, J. O. (1952). Fermentation of 1-C ${ }^{14}$-D-xylose by Lactobacillus pentosus. J. biol. Chem. 194, 555.

Henderson, L. M. \& Snell, E. E. (1948). A uniform medium for determination of amino acids with various microorganisms. J. biol. Chem. 172, 15.

Hills, G. M. (1940). Ammonia production by pathogenic bacteria. Biochem. J. 34, 1057.

Hovgh, L. (1950). Application of paper partition chromatography to the separation of the polyhydric alcohols. Nature, Lond. 165, 400.

Oginsky, E. L. \& Gehrig, R. F. (1952). The arginine dihydrolase system of Streptococcus faecalis. I. Identification of citrulline as intermediate. J.biol. Chem. 198, 791.

Partridge, S. M. (1948). Filter-paper partition chromatography of sugars. I. General description and application to the qualitative analysis of sugars in apple juice, egg white and foetal blood of sheep. Biochem. J. 42, 238.

Peterson, W. H. \& Fred, E. B. (1920). Fermentation of fructose by Lactobacillus pentoaceticus n.sp. J. biol. Chem. 41, 431.

Russell, C., Bhandari, R. R. \& Walker, T. K. (1954). Vitamin requirements of thirty-four lactic acid bacteria associated with brewery products. $J$. gen. Microbiol. 10, 371. 